\title{
Theoretical analysis and experimental demonstration of the radiation mode distribution of $45^{\circ}$ TFG
}

Huabao Qin, Zhikun Xing, Xi Guo, Yarien Moreno, Zhijun Yan, et al.

Huabao Qin, Zhikun Xing, Xi Guo, Yarien Moreno, Zhijun Yan, Qizhen Sun, Kaiming Zhou, Deming Liu, Lin Zhang, "Theoretical analysis and experimental demonstration of the radiation mode distribution of $45^{\circ} \mathrm{TFG}, "$ Proc. SPIE 11048, 17th International Conference on Optical Communications and Networks (ICOCN2018), 1104848 (14 February 2019); doi:

$10.1117 / 12.2522238$

Event: 17th International Conference on Optical Communications and Networks (ICOCN2018), 2018, Zhuhai, China 


\title{
Theoretical analysis and experimental demonstration of the radiation mode distribution of $45^{\circ} \mathrm{TFG}$
}

\author{
Huabao Qin ${ }^{\mathrm{a}, \mathrm{b}}$, Zhikun Xing ${ }^{\mathrm{a}, \mathrm{b}}$, Xi Guo ${ }^{\mathrm{a}, \mathrm{b}}$, Yarien Moreno ${ }^{\mathrm{a}, \mathrm{b}, \mathrm{d}}$, Zhijun Yan ${ }^{\mathrm{a}, \mathrm{b}, \mathrm{c},{ }^{*}}$, Qizhen Sun ${ }^{\mathrm{a}, \mathrm{b}}$, \\ Kaiming Zhou ${ }^{\mathrm{c}}$, Deming Liu ${ }^{\mathrm{a}, \mathrm{b}}$ And Lin Zhang ${ }^{\mathrm{c}}$ \\ aShenzhen Branch of National Engineering Laboratory for NGIA System, Huazhong University of \\ Science and Technology, China; ${ }^{\mathrm{b}}$ The School of Optical and Electronic Information, Huazhong \\ University of Science and Technology, Wuhan, 430074, Hubei, P. R. China; ${ }^{\mathrm{C}}$ Aston Institute of \\ Photonic Technologies, Aston University, Birmingham, UK, B4 7ET; ${ }^{\mathrm{d}}$ Faculty of Electronic and \\ Communication, University of Panama, Republic of Panama. \\ * Corresponding author: yanzhijun@gmail.com
}

\begin{abstract}
In this paper, we have theoretically analyzed and experimentally demonstrated the spatial distribution of the radiation mode of $45^{\circ}$ tilted fiber grating (TFG). The simulation results have shown the intensity distribution of the radiation mode along the fiber axis exhibited an exponential reduction. In experiment, we have observed the radiation mode pattern of a 5-mm long $45^{\circ} \mathrm{TFG}$. The captured profiles along the radial direction and axial direction were in good agreement with the simulated results of the model.
\end{abstract}

Keywords: fiber grating, radiation mode, spatial distribution

\section{INTRODUCTION}

Tilted fiber grating (TFG), which had a special grating structure that was slanted to the fiber axis, was first proposed by Meltz in 1990 [1]. Different from the traditional fiber Bragg grating (FBG) and long period fiber grating (LPFG), the mode coupling inside the TFG was complicated because of the asymmetry structure and exhibited different behaviors. According to the type of mode coupling, TFG could be sorted into three different type: the grating that could couple the forward-propagating core mode into forward-propagating cladding mode and the one that could couple the forward-propagating core mode into backward-propagating cladding mode, and the third kind of TFG was $45^{\circ}$ TFG, which could couple the core mode into the radiation mode [2]. For that the cladding mode was sensitive to the external environment change, the core-cladding mode coupling TFGs had been widely applied in fiber sensing area as some refractive index (RI), liquid level, tension and temperature sensors [3-6]. But for $45^{\circ} \mathrm{TFG}$, it was mostly acted as a polarization element in the fiber optical system because of its unique polarization characteristics. Since that $45^{\circ}$ TFG could couple the s-polarized light of the core mode into radiation mode while remained the ppolarized light travel through the core, it was an ideal in-fiber polarizer that had been applied in all-fiber polarization interference filters and optical fiber lasers [7-8]. Besides, the radiated mode was also attracted researchers' attention and had been applied in spectrally encoded imaging system [9] and optical wireless communication system [10]. However, this applications were only the simple use of the original radiated pattern of $45^{\circ} \mathrm{TFG}$, which had an inherent defect that the energy of the mode was not a uniform distribution and needed to be modified. Unfortunately, although some researches of the radiation mode of $45^{\circ}$ TFG had been carried out, A whole theoretical model for the 3D distribution of the radiation mode of $45^{\circ} \mathrm{TFG}$ was still in blank and the actual radiated pattern of $45^{\circ} \mathrm{TFG}$ was still unknown, which had limited the further development of $45^{\circ}$ TFG.

In this paper, we had theoretically analyzed and improved the existing theoretical model and proposed a 3D theoretical model for analyzing the spatial distribution of the radiation mode of $45^{\circ}$ TFG. In addition, the radiated profile of a 5-mm-long $45^{\circ}$ TFG had been captured, which had experimentally verified our theoretical model.

\section{THEORETICAL MODEL}

A simple and intuitive analysis method for the radiation mode of $45^{\circ} \mathrm{TFG}$ is the volume current method (VCM),

17th International Conference on Optical Communications and Networks (ICOCN2018),

edited by Zhaohui Li. Proc of SPIE Vol. 11048, 1104848 · C 2019 SPIE

CCC code: $0277-786 \mathrm{X} / 19 / \$ 18 \cdot$ doi: $10.1117 / 12.2522238$ 
which has been proposed by Li in 2001 [11]. The VCM regarded the fiber structure as some perturbation of the refractive index, which would induce some perturbed fields with the incidence of the light. And the radiation mode was the result of the induced field. The model based on VCM could properly explain the azimuthal distribution of the radiation mode, which could be written as:

$$
\left.\begin{array}{rl}
\vec{S} & =\frac{\pi c \varepsilon_{0} n_{0}^{2} \delta n^{2} a^{2} k_{0}^{3} E_{0}^{2}}{4 r} F_{1} F_{2}^{2}\left(\hat{r}+\frac{\beta-K_{g}}{k_{t}} \hat{z}\right) \\
F_{1} & =\cos ^{2} \alpha+\sin ^{2} \alpha \sin ^{2}(\delta-\phi) \\
F_{2} & =\frac{K_{s} J_{0}(u a) J_{1}\left(K_{s} a\right)-u J_{1}(u a) J_{0}\left(K_{s} a\right)}{K_{s}^{2}-u^{2}}
\end{array}\right\}
$$

Here $n_{0}$ and $\delta n$ were the refractive index of the fiber core and the modulated index, respectively. $a$ was the radius of the fiber core, $k_{0}$ was wave vector of the incident light, $E_{0}$ was the intensity of the electric field of the incident light, $\beta$ was the propagation constant, $K_{g}=2 \pi \cos \theta / \Lambda$ was the longitudinal wavenumbers of the grating where $\Lambda$ was the period of grating and $\theta$ was the tilted angle, $k_{t}=\sqrt{k_{0}^{2} n_{0}^{2}-\left(\beta-K_{g}\right)^{2}}$ was the projection of the wave vector of emergent light in transverse direction, $\alpha$ and $\phi$ was the emergent angle and the azimuth angle of the of the radiated light, respectively, $\delta$ was the polarization angle, $K_{s}=\sqrt{k_{t}^{2}+K_{t}^{2}-2 k_{t} K_{t} \sin \phi}$ was the mismatch among the wave vectors of incident and emergent and grating and $K_{t}=2 \pi \sin \theta / \Lambda, u$ was waveguide parameter of fiber and $J$ was the Bessel function

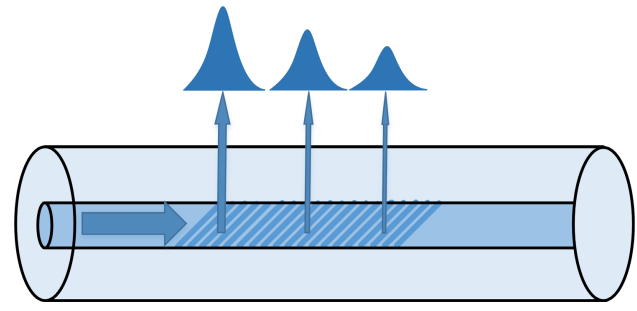

Figure 1. Schematic diagram for analyzing the distribution along the fiber axis.

However, the VCM was not suitable for analyzing the distribution along the fiber because that it was based on the phase-matching condition which only had one radiated direction. In our model, we had divided the grating into many pieces along the fiber, as illustrated in Fig.1, and the whole radiation mode of $45^{\circ}$ TFG was the sum of the radiated pattern of each piece. Hence, with the consideration of the attenuation, the distribution of the radiation mode of $45^{\circ}$ TFG could be expressed as:

$$
I=\alpha(1-\alpha)^{\frac{L}{\Lambda}-1} I_{0}
$$

Where $I$ was the radiated intensity and $I_{0}$ was the intensity of the incident light. $L$ and $\Lambda$ were the length and the period of the grating, respectively. $\alpha$ was the coupling efficiency between the core mode and the radiation mode. Eq.2 described the relationship of the radiated intensity and the grating length, which indicated the distribution of the mode along the fiber axis. And the combination of Eq.1 and Eq.2 was the model that we proposed to describe the distribution of the radiation mode of $45^{\circ} \mathrm{TFG}$.

\section{SIMULATED AND EXPERIMENTAL RESULTS}

For a $45^{\circ} \mathrm{TFG}$ with the certain structure parameters, the distribution of the radiation mode could be easily figure out by applying the model. The simulated results of the radiation mode of a $5-\mathrm{mm}$ long $45^{\circ} \mathrm{TFG}$ was showed in Fig.2. It was clearly showed in the figure that the intensity of the mode reached maximum at the beginning of the grating and gradually decreased with the increase of the grating length. The azimuthal distribution of the mode had a Gaussianlike profile with the energy mainly distributed in about $20^{\circ}$ azimuthal angle range from $80^{\circ}$ to $100^{\circ}$ and the divergence angle of the mode was $12^{\circ}$, where the maximum intensity was appeared at $90^{\circ}$. 


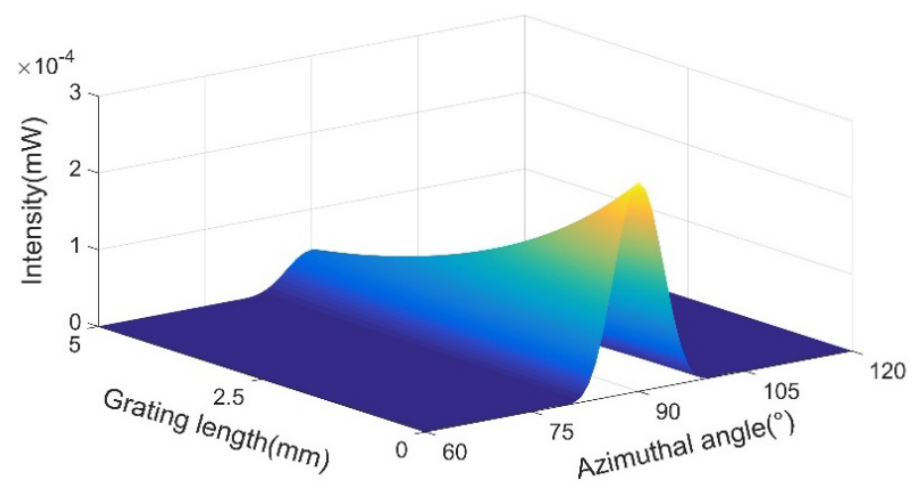

Figure 2. Simulated 3D results of the spatial distribution of the radiation mode.

In addition, to verify our model, we had fabricated a 5-mm long $45^{\circ} \mathrm{TFG}$ and set up a measuring system to capture the radiated pattern, which was illustrated in Fig.3a. In the setup, the tunable laser was used to generate a $1550 \mathrm{~nm}$ linear polarized light, the polarization controller (PC) and the powermeter were used to adjust the polarization state of the light that was incident to the grating. The beam profile was used to capture the intensity pattern of the radiation mode of the grating.

(a)

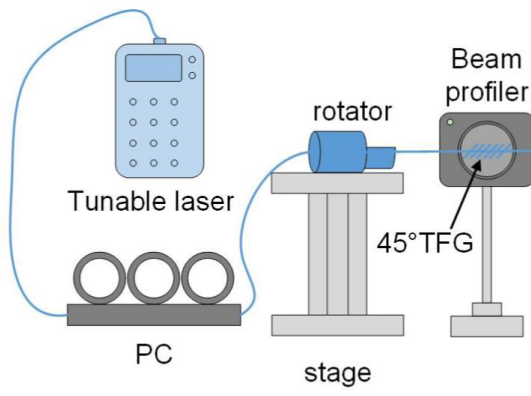

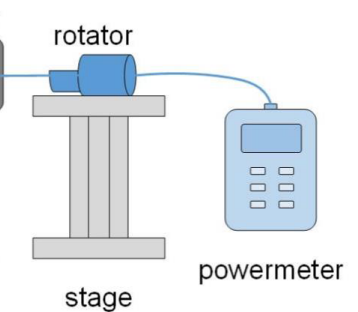

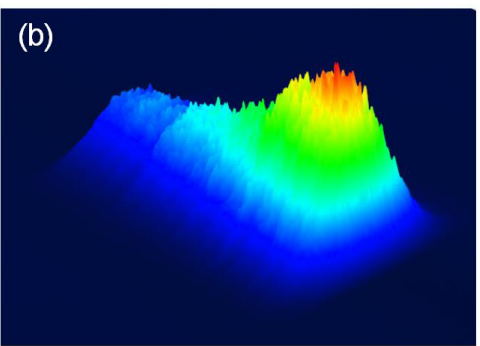

Figure 3. (a) the experimental setup for capturing the radiation mode; (b) the captured radiation pattern

The detailed image of the actual radiation mode pattern was shown in Fig.3b, and the azimuthal distribution and the distribution along the fiber axis were shown in Fig.4a and Fig.4b, respectively. Compared with the simulated results, the radiated pattern had a similar profile where the highest intensity occurred at the beginning of the grating and reduced in the form of an exponential function. The divergence angle of the mode was about $12.18^{\circ}$ and the length of the pattern along the axial direction was about 5068um, which was in accord with the actual grating length. As shown in Fig.4a and Fig.4b, the simulated results were in a good agreement with the experimental results, demonstrating the reliability of the $3 \mathrm{D}$ model we proposed. The unsmooth profile of the pattern was caused by the noise of the sensor.
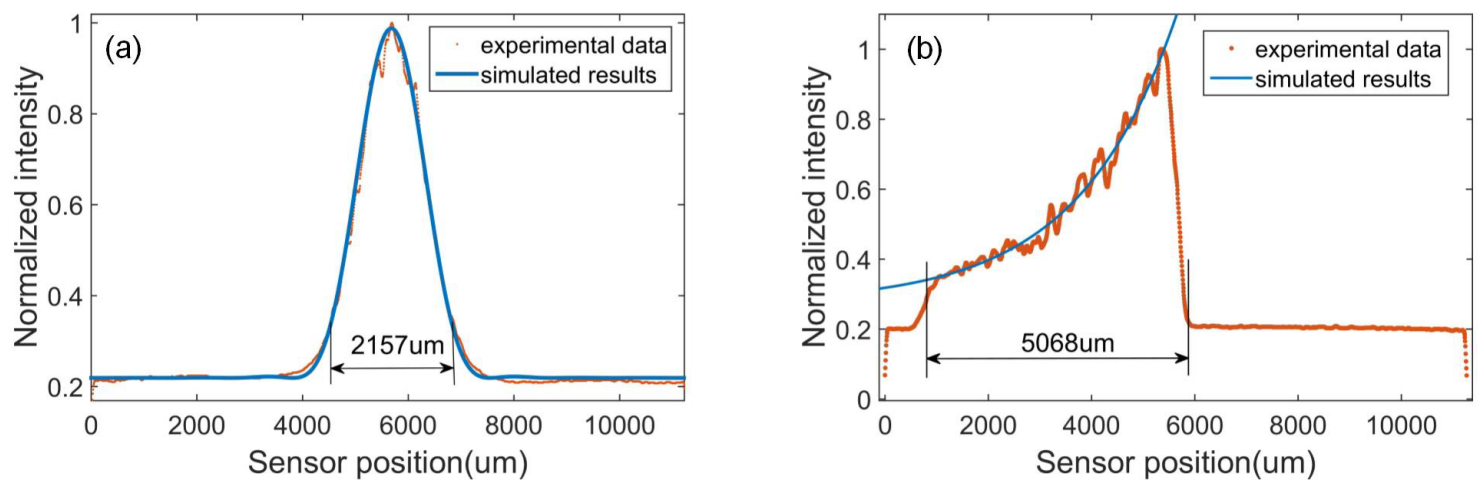

Figure 4. The actual distribution of the radiation mode and the simulated results in (a) radial direction; (b) axial direction 


\section{CONCLUSION}

In conclusion, we had proposed a three-dimension theoretical model for the distribution of the radiation mode of $45^{\circ} \mathrm{TFG}$. The intensity distribution of the radiation mode along the fiber axis had been analyzed and figured out, which showed an exponential reduction in the axial direction. A 5-mm long $45^{\circ} \mathrm{TFG}$ had been fabricated and the radiated pattern had been captured with a designed experimental setup. For a uniform $45^{\circ} \mathrm{TFG}$, the whole distribution of the radiation mode was unbalanced, where the energy was mainly distributed at the beginning of the grating, while this unbalanced distribution of energy had some inherent defects. The proposed model could be used to modified the mode

and obtain a desire radiated pattern, which would benefit the applications of $45^{\circ} \mathrm{TFG}$ in imaging, spectrum analysis and optical wireless communication system.

\section{Acknowledgement:}

This work is supported by Shenzhen Development and Reform Commission (No. (2017)853), National Natural Science Foundation of China (No. 61505244), the Science Fund for Creative Research Groups of the Nature Science Foundation of Hubei (NO. 2018CFA004) and the Major Projects of Technical Innovation of Hubei (NO. 2018AAA040).

\section{REFERENCES}

[1] G. Meltz, W. Morey, and W. Glenn, "In-fiber Bragg grating tap," Optical Fiber Communication Conference, TUG1 (1990).

[2] Z. Yan, K. Zhou, and L. Zhang, "In-fiber linear polarizer based on UV-inscribed $45^{\circ}$ tilted grating in polarization maintaining fiber," Opt. Lett. 37(18), 3819-3821 (2012).

[3] B. Jiang, K. Zhou, C. Wang, Y. Zhao, J. Zhao, and L. Zhang, "Temperature-calibrated high-precision refractometer using a tilted fiber Bragg grating," Opt. Express 25(21), 25910-25918 (2017).

[4] B. Gu, W. Qi, Y. Zhou, Z. Wu, P. P. Shum, and F. Luan, "Reflective liquid level sensor based on modes conversion in thin-core fiber incorporating tilted fiber Bragg grating," Opt. Express 22(10), 11834-11839 (2014).

[5] Z. Yan, Q. Sun, C. Wang, Z. Sun, C. Mou, K. Zhou, D. Liu, and L. Zhang, "Refractive index and temperature sensitivity characterization of excessively tilted fiber grating," Opt Express 25(4), 3336-3346 (2017).

[6] C. Shen, C. Zhong, D. Liu, X. Lian, J. Zheng, J. Wang, Y. Semenova, G. Farrell, J. Albert, and J. F. Donegan, "Measurements of milli-Newton surface tension forces with tilted fiber Bragg gratings," Opt. Lett. 43(2), 255-258 (2018).

[7] Z. Yan, H. Wang, K. Zhou, Y. Wang, W. Zhao, and L. Zhang, "Broadband tunable all-fiber polarization interference filter based on 45 tilted fiber gratings," J. Lightwave Technol. 31(1), 94-98 (2013).

[8] C. Zou, T. Wang, Z. Yan, Q. Huang, M. AlAraimi, A. Rozhin, and C. Mou, "Wavelength-tunable passively modelocked Erbium-doped fiber laser based on carbon nanotube and a 45 tilted fiber grating," Opt. Commun. 406(151157 (2018).

[9] G. Wang, C. Wang, Z. Yan, and L. Zhang, "Highly efficient spectrally encoded imaging using a $45^{\circ}$ tilted fiber grating," Opt. Lett. 41(11), 2398-2401 (2016).

[10] G. Wang, U. Habib, Z. Yan, N. J. Gomes, Q. Sui, J. Wang, L. Zhang, and C. Wang, "Highly efficient optical beam steering using an in-fiber diffraction grating for full duplex indoor optical wireless communication," J. Lightwave Technol. (2018).

[11] Y. Li, M. Froggatt, and T. Erdogan, "Volume current method for analysis of tilted fiber gratings," J. Lightwave Technol. 19(10), 1580-1591 (2001). 